\title{
Analysis
}

\section{Mobile Health Approaches to Non-Communicable Diseases in Rwanda}

\author{
Brenda Asiimwe-Kateera ${ }^{1 *}$, Jeanine Condo ${ }^{1}$, Albert Ndagijimana ${ }^{1}$, Sanjeev Kumar ${ }^{2}$, \\ Madeleine Mukeshimana ${ }^{1}$, Eric Gaju ${ }^{3}$, Andrew Muhire ${ }^{3}$, Marie Aimee Muhimpundu ${ }^{4}$, Mi Ja Kim ${ }^{1}$, \\ Ann Kurth ${ }^{5}$ \\ ${ }^{1}$ College of Medicine and Health Sciences, University of Rwanda, Kigali, Rwanda \\ ${ }^{2}$ Department of Health Policy and Management, Yale School of Public Health, .New Haven, CT, US A \\ ${ }^{3}$ Ministry of Health, Kigali, Rwanda \\ ${ }^{4}$ Non-Communicable Diseases Division, Rwanda Biomedical Center, Kigali, Rwanda \\ ${ }^{5}$ New York University, College of Nursing, and NYU Global Institute of Public Health, New York, NY, USA
}

\begin{abstract}
Rwanda is affected by a substantial dual burden of a rapid epidemiological rise in non-communicable diseases (NCDs) against the backdrop of high infectious disease rates. The Global Burden of Disease study showed that premature deaths due to NCDs such as diabetes and hypertension are increasing, accounting for $30 \%$ of all deaths in Rwanda in 2010. The usefulness of $\mathrm{mHealth}$ interventions has been shown for reducing adverse effects of diabetes and hypertension. Because Rwanda RapidSMS system is already successfully operating in maternal, neonatal and child health, it would be cost-effective to leverage this infrastructure and adapt it for the NCD domain. However, rigorous evaluations of the efficacy of $\mathrm{mHealth}$ intervention in the area of NCDs are limited. Hence, developing a robust mHealth intervention study is urgent through a contextual combination of quantitative and qualitative study designs. This paper highlights the significance of the problem of NCDs, usefulness of mHealth interventions for stemming diabetes and hypertension, and an urgent need for research on using mHealth interventions for prevention of NCDs in Rwanda. Carefully conducted research on the efficacy and effectiveness of mHealth intervention will have ramifications for the evidence based policy, decision making, practice and research in other LMICs.
\end{abstract}

Key words: NCDs, Diabetes, Hypertension, mHealth intervention, Africa, Rwanda

\section{Introduction}

Non-communicable diseases (NCDs) are a global phenomenon, affecting increasing numbers of individuals in low and middle-income countries (LMICs). NCDs have become a leading cause of death affecting populations across ages and environments. In 2008, NCDs contributed to $63 \%$ of the 57 million deaths worldwide(Alwan, 2010). The growing incidence of NCDs is a matter of grave concern - a concern that was previously reserved largely for older and richer individuals. However, a high proportion of younger people are increasingly affected in LMICs. These diseases include cancer, chronic respiratory diseases, cardiovascular diseases, especially an early onset of hypertension, type I and II diabetes, and often neglected oral and mental health (Beaglehole et al., 2011)principally heart disease, stroke, cancer, diabetes, and chronic respiratory diseases, are a global crisis and require a global response. Despite the threat to human development, and the availability of affordable, cost-ef- fective, and feasible interventions, most countries, development agencies, and foundations neglect the crisis. The UN High-Level Meeting (UN HLM).

Diabetes and cardiovascular diseases are among the most frequently occurring NCDs in Rwanda with an increasing prevalence of other chronic NCDs such as cancer, chronic respiratory diseases, injuries and disabilities(Ministry of Health, 2012). These chronic NCDs in Rwanda accounted for an estimated $29 \%$ of all mortalities in 2008 (Commonwealth Health Online, 2015). The speed, scale, and consequences of the increase in NCDs are worrisome, especially in light of an abysmally low quality of health system infrastructure, poor health literacy, and endemic poverty in many LMICs. In the wake of the rise of NCD-related morbidity in Rwanda, especially among younger populations, the Rwandan government has committed to decrease NCD-related deaths by $80 \%$ among people under 40 years of age by 2020 through the implementation of population-level interventions(Binagwaho, Muhimpundu, \& Bukhman, 2014). The immediate

*Correspondence: bkateera@gmail.com 
need in Rwanda is to sensitize the population about death and disability due to NCDs and to develop innovative approaches to use existing resources and technology in a cost-effective manner.

\section{Harnessing existing infrastructure and resources}

Rwanda has a well-functioning health care referral system starting with community healthcare workers (CHWs), who communicate and facilitate patient referrals to health posts and/or health centers, and then to hospitals. CHWs are often the first point of contact with the health care system; each village has three $\mathrm{CHWs}$ who have been trained to identify and address common conditions. All $\mathrm{CHWs}$ have been given mobile phones to enable real-time reporting on all community health data from community to the central level system tracking the 1,000 days of life (pregnancy until birth, and newborn until 2 years) using RapidSMS, an mHealth technology. Since the Rwanda RapidSMS system is already successfully operating in maternal, neonatal and child health $(\mathrm{MNCH})$, it would be efficient and cost-effective to leverage this infrastructure and adapt it for the NCD domain(Ngabo et al., 2012).

The Rwanda Ministry of Health $(\mathrm{MoH})$ Health Information System (HIS) also provides key infrastructure that could be adapted to support NCD reporting and interventions. HIS software is an open-source platform that can be expanded for other uses, such as intelligent patient based monitoring and increased reporting frequency. The current Rwanda MoH HIS contains an NCD module and NCD indicators that could be added into the CHW reporting system since the HIS platform supports SMS reporting technology that the CHWs use.

\section{Use of mHealth for public health interventions}

The majority of NCD risk factors are preventable through a broad range of cost-effective public health interventions that can be delivered by CHWs or health posts via various means including the use of mHealth technology. The mHealth field is accruing evidence that technology integration within the health sector has great potential to promote better health communication to influence positive healthy lifestyles, improve decision-making by health professionals and clients, and enhance healthcare quality by improving access to health information and facilitating instant communication where this was not previously possible(Cole-Lewis \& Kershaw, 2010)inexpensive, and instant. This systematic review provides an overview of behavior change interventions for disease management and prevention delivered through text messaging. Evidence on behavior change and clinical outcomes was compiled from randomized or quasi-experimental con- trolled trials of text message interventions published in peer-reviewed journals by June 2009. Only those interventions using text message as the primary mode of communication were included. Study quality was assessed by using a standardized measure. Seventeen articles representing 12 studies (5 disease prevention and 7 disease management. Ideally mHealth should be used to augment the productivity of CHWs and nurses who are the backbone of healthcare in Rwanda.

MHealth has been widely used in developed countries to prevent diseases and promote healthy lifestyles among community members, through the communication modalities of text message services (SMS), voice calling, or email in line with the chronic care model(Fig. 1) (Coleman, Austin, Brach, \& Wagner, 2009). In the context of poorly-developed health systems and poverty in LMICs, Kahn et al. (2010) affirmed that mHealth could potentially provide the greatest impact, facilitating health-related information in an efficient and cost-effective way(Kahn, Yang, \& Kahn, 2010). However, to date too few mHealth interventions in LMICs have focused on NCDs such as diabetes or hypertension(Cole-Lewis \& Kershaw, 2010; Gurman, Rubin, \& Roess, 2012; Thirumurthy \& Lester, 2012).

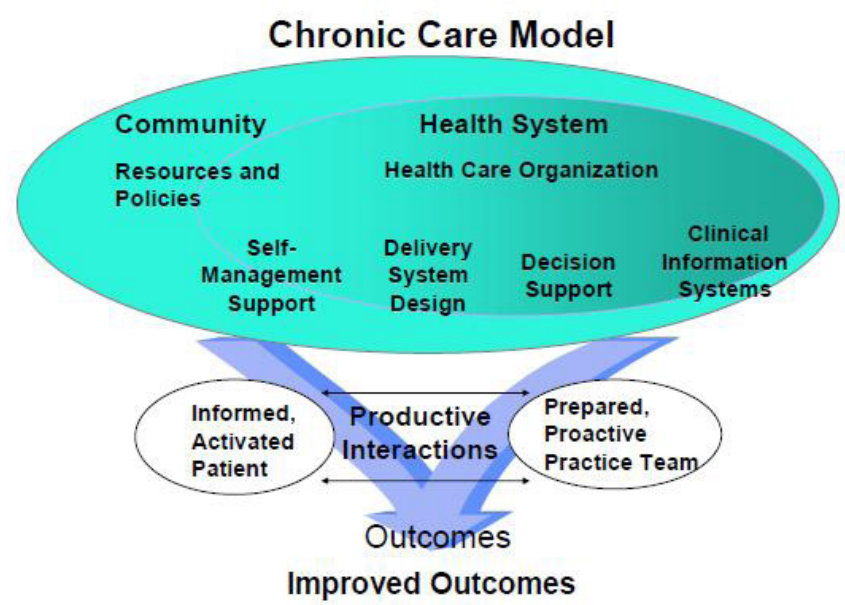

Figure 1. The Chronic Care Model

There is ample evidence in the literature that demonstrates the usefulness of ICT tools such as mHealth interventions (e.g., telemedicine, Web-based strategies, e-mail, mobile phones, mobile applications, text messaging, and monitoring sensors) for reducing adverse effects of diabetes and hypertension in developed countries(de Jongh, Gurol-Urganci, Vodopivec-Jamsek, Car, \& Atun, $2012 \mathrm{~b})$. This suggests the need for testing combinations of mHealth tools that are contextualized to local issues in LMICs and taking into consideration the perceived 
usefulness and ease of use as outlined in the Technology Acceptance Model(Kim \& Park, 2012)it is mandatory that health consumers have the behavioral intention to measure, store, and manage their own health data. Understanding health consumers' intention and behavior is needed to develop and implement effective and efficient strategies. $\backslash \mathrm{n} \backslash \mathrm{nOBJECTIVE:} \mathrm{To} \mathrm{develop} \mathrm{and} \mathrm{verify} \mathrm{the}$ extended Technology Acceptance Model (TAM. A meta-analyses by Widmer et al.found that mHealth tools reduced cardiovascular outcomes, obesity/BMI. (Widmer et al., 2015) A Cochrane review (de Jongh 2012) found that SMS based interventions had an impact on hypertension treatment compliance as well as glucose monitoring among diabetics, suggesting that these tools can support self-management of long-term illnesses. They did caution, however, that there are "significant information gaps regarding the long-term effects, acceptability, costs, and risks of such interventions.'(de Jongh, Gurol-Urganci, Vodopivec-Jamsek, Car, \& Atun, 2012a). Mobile phone interventions for diabetes self-management in a meta-analysis of 22 studies with 1,657 participants showed reduced $\mathrm{HbA}(1 \mathrm{c})$ values by a mean of $0.5 \%$ [6 mmol $/ \mathrm{mol} ; 95 \%$ CI $0.3-0.7 \%$ ] over a median of 6 months follow-up duration (Liang et al.). The effect was stronger among individuals with Type 2 rather than Type 1 diabetes $(\mathrm{P}=0.02)$ (Liang et al., 2011).

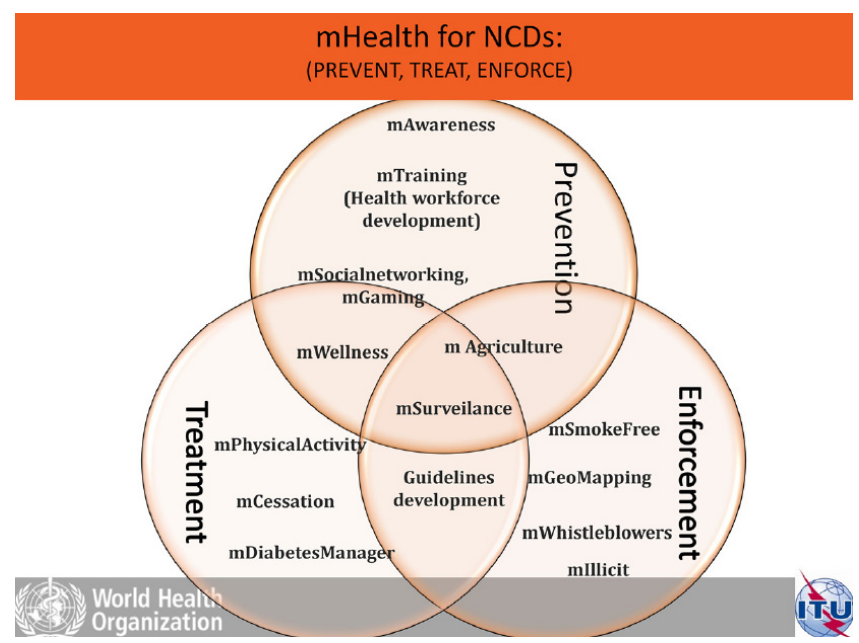

Figure 2. WHO mHealth for NCD Model

Source: WHO and ITU, 2012

WHO has pointed out that a number of countries have piloted the use of mHealth against the NCD epidemic with successful outcomes. These have included messaging on health promotion, NCD risk factors and how to change unhealthy behaviors and assist with implementing national laws on NCDs. A growing criticism is that most mHealth projects have been analyzed for pilot feasibility rather than for their efficacy and effectiveness in improving health outcomes(Tomlinson, Rotheram-Borus,
Swartz, \& Tsai, 2013). For those few studies doing impact assessments, the research designs are not usually robust. These "successful pilots" should be scaled up and the framework in Figure 2 may be useful for that purpose.

In particular, mobile phones have been a primary tool in mHealth approaches, being used for data capture and communication by public health and health care workers for surveillance, screening, and point of care diagnosis as well as communication with clients for prevention and healthy behavior and medication adherence. Currently Rwanda has achieved a mobile phone penetration of $70 \%$ with higher coverage in urban areas (RURA, 2015). Rwanda also has been a leader in identifying $m$ Health strategies for MCNH through RapidSMS (mHealth Alliance, 2013) yet; its extension to NCDs is under developed. No program currently exists in Rwanda that simultaneously addresses prevention of diabetes and hypertension using community level and mobile technological interventions.

\section{Models for NCD prevention}

We believe that primary, secondary and tertiary adapted prevention models could be implemented in Rwanda facilitated with mobile technology on behavior change in the following ways:

At the first level, customized messages about risk factors and a healthy lifestyle for people at risk should be created and shared with health care providers taking care of the clients in the RapidSMS and linked with national HIS. At the second level, CHWs at the village level would deliver modules on prevention to the entire population at community meetings. During the meetings, participants would be offered basic screening including measures such as blood pressure, blood glucose, and BMI (body weight and height). At the third level, the CHWs would identify the population at risk and refer them to the nearest health post/health center for more tests and follow up. This level 3 will refer complicated cases to level 4 where district hospitals will ensure proper management of complicated cases of NCDs. Using the triple-prevention model using 3 levels will not only end up sensitizing the whole Rwandan health care system about the NCD burden but will also prepare it to address NCDs at individual, family, and community levels. Given the constraints regarding health care workers shortages in many LMICS, an effective and judicious use of mHealth interventions will help stem the spread of NCDs in their early stages.

\section{Conclusion}

There is an urgent need for the research community to invest time and resources to develop rigorous mHealth intervention studies or implementation research through 
a contextual combination of quantitative and qualitative study designs. The new or updated mHealth system would address burden disease, especially chronic diseases that traditional health system could not address. This is essential for optimally scaling up the promises and opportunities inherent in the mHealth technology, Lessons learned from rigorously conducted studies on the efficacy and cost-effectiveness of mHealth intervention in Rwanda will have ramifications for evidence based policy, decision making, practice and research in other LMICs.

\section{Authors' contributions}

MJK, AK, BAK, JC conceived the idea for the manuscript, JC,BAK, AN, SK, MM, EG, AM, MAM, MJK and $\mathrm{AK}$ reviewed and provided substantial edits. All authors approve the final version.

\section{Acknowledgements}

We thank Nok Chhun and Beth Shair at NYU College of Nursing for their contributions and support.

\section{Funding support: None}

\section{Conflict of interest}

All authors report no conflict of interest.

\section{References}

Alwan, A. (2010). Global status report on noncommunicable diseases. World Health Organization, 176. doi:978 924156422 9

Beaglehole, R., Bonita, R., Alleyne, G., Horton, R., Li, L., Lincoln, P., Stuckler, D. (2011). UN high-level meeting on non-communicable diseases: Addressing four questions. The Lancet. doi:10.1016/S0140-6736(11)60879-9

Binagwaho, A., Muhimpundu, M. A., \& Bukhman, G. (2014). 80 under 40 by 2020: an equity agenda for NCDs and injuries. The Lancet, 383(9911), 3-4.

Cole-Lewis, H., \& Kershaw, T. (2010). Text messaging as a tool for behavior change in disease prevention and management. Epidemiologic Reviews, 32(1), 56-69. doi:10.1093/epirev/ mxq004

Coleman, K., Austin, B. T., Brach, C., \& Wagner, E. H. (2009). Evidence on the Chronic Care Model in the new millennium. Health Affairs (Project Hope), 28(1), 75-85. doi:10.1377/ hlthaff.28.1.75

Commonwealth Health Online. (2015). Non-communicable diseases in Rwanda.
De Jongh, T., Gurol-Urganci, I., Vodopivec-Jamsek, V., Car, J., \& Atun, R. (2012a). Mobile phone messaging for facilitating self-management of long-term illnesses. The Cochrane Library.

De Jongh, T., Gurol-Urganci, I., Vodopivec-Jamsek, V., Car, J., \& Atun, R. (2012b). Mobile phone messaging for facilitating self-management of long-term illnesses. The Cochrane Database of Systematic Reviews, 12, CD007459. doi:10.1002/14651858.CD007459.pub2

Gurman, T. A., Rubin, S. E., \& Roess, A. A. (2012). Effectiveness of mHealth Behavior Change Communication Interventions in Developing Countries: A Systematic Review of the Literature. Journal of Health Communication. doi:10.1080/10 810730.2011.649160

Kahn, J., Yang, J., \& Kahn, J. (2010). "Mobile” Health Needs And Opportunities In Developing Countries. Health Affairs, 2(2), 252-258. doi:10.1377/hlthaff

Kim, J., \& Park, H.-A. (2012). Development of a health information technology acceptance model using consumers' health behavior intention. Journal of Medical Internet Research, 14(5), e133. doi:10.2196/jmir.2143

Liang, X., Wang, Q., Yang, X., Cao, J., Chen, J., Mo, X., Gu, D. (2011). Effect of mobile phone intervention for diabetes on glycaemic control: a meta-analysis. Diabetic Medicine, 28(4), 455-463.

mHealth Alliance. (2013). mHealth Alliance Honors Mobile Health Visionaries in First-Ever Awards Ceremony at mHealth Summit. Retrieved from http://www.prweb.com/ releases/2013/12/prweb11406321.htm

Ministry of Health. (2012). Health Sector strategic Plan July 2012-June 2018, (July), 170.

Ngabo, F., Nguimfack, J., Nwaigwe, F., Mugeni, C., Muhoza, D., Wilson, D. R., Binagwaho, A. (2012). Designing and Implementing an Innovative SMS-based alert system (RapidSMS-MCH) to monitor pregnancy and reduce maternal and child deaths in Rwanda. The Pan African Medical Journal, 13.

RURA. (2015). Rwanda Monthly Telecommunications Subscriptions Report.

Thirumurthy, H., \& Lester, R. T. (2012). M-health for health behaviour change in resource-limited settings: Applications to HIV care and beyond. Bulletin of the World Health Organization, 90(5), 390-392. doi:10.2471/BLT.11.099317

Tomlinson, M., Rotheram-Borus, M. J., Swartz, L., \& Tsai, A. C. (2013). Scaling Up mHealth: Where Is the Evidence? PLoS Medicine, 10(2), 1-5. doi:10.1371/journal.pmed.1001382

Widmer, R. J., Collins, N. M., Collins, C. S., West, C. P., Lerman, L. O., \& Lerman, A. (2015). Digital Health Interventions for the Prevention of Cardiovascular Disease: A Systematic Review and Meta-analysis. In Mayo Clinic Proceedings (Vol. 90, pp. 469-480). 